\title{
MODELAGEM DA DESRAMA NATURAL DE Araucaria angustifolia (BERT.) O. KTZE
}

\author{
Leif Nutto ${ }^{1}$
}

Peter Spathelf ${ }^{2}$

\begin{abstract}
RESUMO
Neste trabalho foi analisada a dinâmica e a qualidade da desrama natural de Araucaria angustifolia (Bert.) O. Ktze. em diferentes sítios no Sul do Brasil, dentro de uma estratégia da produção de madeira de alta qualidade. O material usado se constitui de 400 árvores de florestas nativas e plantadas, crescendo sob variadas condições e tratamentos. $\mathrm{O}$ método usado foi a análise exploratória dados levantados de parcelas temporárias. Foram feito mapas de distribuição das árvores e das projeções das copas para avaliar a competição e o seu efeito na desrama. $\mathrm{O}$ estudo mostra que existe uma relação estreita entre o crescimento em altura e diâmetro, numa idade determinada, e a inserção da copa. O primeiro galho morto está estreitamente ligado com a inserção da copa. Estas variáveis foram usadas para modelar o desenvolvimento da inserção da copa e da altura comercial ao longo do tempo. Além disso, podem ser derivados destes modelos a dimensão do núcleo enodado. Foi mostrado que para o manejo de araucaria em rotações curtas uma poda artificial aumenta significativamente à parte da madeira valiosa sem nós.

Palavras-chave: poda, núcleo enodado, madeira de qualidade, manejo
\end{abstract}

\section{MODELLING SELF-PRUNING OF ARAUCARIA ANGUSTIFOLIA (BERT.) O. KTZE}

\begin{abstract}
In the present study the self-pruning dynamics and quality of Araucaria angustifolia (Bert.) O Ktze. on different sites in Southern Brazil was analyzed in the framework of a strategy of high quality wood production. The material consisted of 400 trees from native forests and plantations growing under varying site conditions and treatments in the Brazilian states of Santa Catarina, Parana and Rio Grande do Sul. The research method applied was an explorative analysis of data taken from temporary sample plots. For each plot stem distribution and crown projection map was established in order to determine the competition status and its impact on selfpruning. The study shows that diameter and height at a certain age are good estimators for predicting height of crown base. The lowest dead branch is closely related to crown base. These parameters are used to model the dynamics of self-pruning depending on radial increment and height growth. Moreover, the dimension of the knotty core was calculated. It could be shown that Araucaria angustifolia has a retarded self-pruning because of its tendency of maintaining dead branches at the stem. Especially for target diameters of 40 to $50 \mathrm{~cm}$ within short rotation periods an artificial pruning is recommended in order to reduce the knotty core.
\end{abstract}

Key words: knot-free core, high quality timber, silviculture

\section{INTRODUÇÃO}

No passado as matas de araucária caracterizaram 20 milhões de hectares da paisagem no Sul do Brasil. A crescente necessidade de terras para a agricultura e pecuária, como também as amplas possibilidades de utilização do Pinheiro-doParaná, têm conduzido, desde a metade do século passado, à exploração e destruição destas matas. Das florestas nativas de Araucaria angustifolia sobraram, em 1980,

\footnotetext{
${ }^{1}$ Engenheiro Florestal, Dr., Pesquisador Senior, CIS-Madera, Parque Tecnolóxico da Galicia, Avda. Da Galicia nº 5, San Cibrao das Viñas, E-32901 OURENSE, Espanha; e-mail: nutto@cismadera.com

${ }^{2}$ Engenheiro Florestal, Dr., Forstdirektion Tübingen, Im Schloss, D-72074 Tübingen, Alemanha; e-mail: Peter.Spathelf@forst.bwl.de *O trabalho foi realizado na Universidade Federal de Santa Maria, RS, quando os autores eram Pesquisador Visitante do DAAD e Professor Visitante da CAPES/DAAD respectivamente.
}

Recebido para publicação: 03/02/2003

Aceito para publicação: 11/09/2003 
menos de 3\% (Machado e Siqueira, 1980). As espécies competindo com a araucária na sua área de ocorrência natural são do gênero Eucalyptus e Pinus. Essas possuem um potencial de crescimento volumétrico superior ao da araucária, especialmente em sítios de baixa produtividade. Para aumentar o interesse econômico da araucária, novos objetivos e formas de manejo devem ser considerados. Uma opção poderia ser a produção de madeira de alta qualidade com um preço por metro cúbico superior ao de pinus ou eucalipto.

Os parâmetros mais importantes que podem ser influenciados pelo manejo com o objetivo de produzir madeira de alta qualidade para serraria, painéis ou laminação, são a dimensão e a galhosidade interna do fuste (Hawley e Smith, 1972). O objetivo de produzir maiores dimensões pode levar a rotações mais longas. Como alternativa poderse-ia, também, promover desbastes fortes com a finalidade de aumentar o tamanho da copa e assim acelerar o crescimento em diâmetro (Nutto, 2000). A ausência de nós, pelo menos na primeira parte do fuste, pode ser alcançada por uma desrama natural ou artificial. Várias espécies florestais, sobretudo folhosas, têm a característica de facilmente perderem os seus galhos, sendo, consequentemente, apropriadas para uma estratégia de condução que incorpora a desrama natural. Nas coníferas, no entanto, os galhos mortos ainda permanecem na árvore um longo tempo, e uma boa parte dos mesmos é incluída no fuste, causando um núcleo enodado de grande dimensão (Mayer-Wegelin, 1936).

Quando um galho é sombreado pela copa acima e pelos concorrentes vizinhos, ele reduz a produção fotossintética e logo é desconectado do sistema de abastecimento de assimilatas de partes da copa superior. Daqui em diante ele depende somente da produção própria e não contribui mais ao crescimento em diâmetro da árvore. Com a intensidade de luz diminuindo, o galho começa a morrer dentro de pouco tempo. Os galhos da parte baixa da copa até reduzem o crescimento em diâmetro (Mitscherlich e Von Gadow, 1968). Isto pode ser explicado com o consumo de água e nutrientes na respiração destes galhos, sem contribuir para o crescimento da árvore (Kozlowski, 1971).

No ambiente natural a ineficiência assimilatória ou danos abióticos e bióticos levam à perda dos galhos pela árvore. As árvores possuem mecanismos fisiológicos para reagir a esses traumas, através de reações químicas nas células do tronco e da base do galho, que criam barreiras impedindo a entrada de organismos degradadores no lenho do tronco. Este processo é chamado 'compartimentalização' (Shigo, 1979; Schilling, 1996). A maioria das coníferas formam bolsas de resina sobre os tocos dos ramos desramados, principalmente nos casos de cicatrização lenta. As feridas criadas ao extrair galhos vivos cicatrizam mais rapidamente do que as que se formam ao podar galhos mortos e secos, já que quando se corta madeira viva o câmbio ao redor do toco já está em posição para formar calo (Mayer-Wegelin, 1936). Mas no caso de uma poda de galhos ainda vivos, a árvore não tem a possibilidade de iniciar a criação de barreiras antecipadas, que impediriam a entrada de organismos danificadores, o que pode causar podridão na madeira do fuste.

Apesar da araucária pertencer ao grupo com processo de desrama natural lenta, a dinâmica da desrama natural é considerada boa, comparada com a de outras coníferas. Mesmo assim, para a produção de madeira de alto valor, uma poda artificial é aconselhável (Seitz, 1986), especialmente considerando os diâmetros-objetivo de somente 40 a $50 \mathrm{~cm}$.

Portanto, para otimizar a produção de madeira de alta qualidade, é de grande importância conhecer os impactos de diferentes tratamentos silviculturais no processo da desrama natural. Um programa desses requer o conhecimento de variáveis baseadas na árvore individual. $\mathrm{O}$ desenvolvimento do diâmetro de uma árvore está estreitamente ligado à expansão da copa. Dessa forma, um crescimento rápido em diâmetro pode ser apenas sustentado com uma expansão mais rápida da copa. Isso leva a galhos mais longos e grossos para suportar a crescente massa foliar. Para algumas espécies do gênero Picea, Populus e Quercus, foi encontrada uma relação estreita entre a espessura dos galhos na inserção no tronco e o seu comprimento (Deleuze et al., 1996; Nelson et al., 1981; Nutto, 1999). Portanto, uma rápida expansão da copa retarda o processo de desrama natural do fuste (Spiecker, 1991; Nutto, 1999), ou seja, para restringir o núcleo enodado de um tronco, é 
necessário manter uma alta densidade inicial do povoamento (Nutto e Spiecker 2000). A estratégia de forçar a desrama natural com alta densidade inicial de um povoamento é chamada de 'qualificação', levando a um coletivo de árvores com fustes limpos de nós antes da fase de desbaste (Spiecker 1991).

O gênero Araucaria da família Araucariaceae só ocorre no hemisfério Sul, com duas das 14 espécies na América do Sul (Whitmore, 1975), sendo a Araucaria angustifolia (Bert.) O. Ktze. a espécie de maior importância no mercado mundial. Isso pode ser reconhecido pelos nomes que existem em diferentes países: Paraná pine (Inglês), Brasilkiefer (Alemão), Araucaria du Brésil (Francês), Pino misionero (Espanhol) e Pinheiro-do-Paraná (Português) (Lamprecht, 1986). Por causa da ameaça de extinção, o volume de exportação de araucária foi contingenciado pelo IBAMA (1998). Entre 1995 e 1998, somente um volume de 52.000 $\mathrm{m}^{3}$ por ano foi liberado para a exportação.

A região da ocorrência natural se limita entre as latitudes de $15^{\circ}$ e $30^{\circ} \mathrm{Sul}$, e longitudes de $43^{\circ}$ e $57^{\circ}$ Oeste, com maior distribuição nos estados de Rio Grande do Sul, Santa Catarina e Paraná, e menores áreas na província de Misiones (Argentina) e nos estados de São Paulo, Minas Gerais e Rio de Janeiro (Hueck, 1972).

A araucária se encontra em altitudes entre 600 e $800 \mathrm{~m}$, subindo até 1200 e $1800 \mathrm{~m}$ na Serra da Mantiqueira. O clima na área de ocorrência natural é classificado como temperado, no qual a temperatura média anual varia de 13 a $18^{\circ} \mathrm{C}$, caraterizados por verões frescos e invernos relativamente frios até $-8^{\circ} \mathrm{C}$, mas com baixa freqüência de geadas (Golfari, 1967).

$\mathrm{Na}$ sua forma atual, o manejo de plantações da araucária se constitui na condução de povoamentos puros com uma idade de rotação de até 40 anos. A madeira é apropriada para celulose, construção civil e laminação. No Sul do Brasil, povoamentos de Araucaria angustifolia mostram incrementos médios anuais em torno de $10-25 \mathrm{~m}^{3}$ por ano por hectare.
Em vista disso, o presente trabalho tem como objetivo determinar, analisar e modelar as relações entre a desrama natural da Araucaria angustifolia e o crescimento em diâmetro e em altura, como estratégia de produção de madeira de alta qualidade.

\section{MATERIAL E MÉTODOS}

\section{Hipóteses de trabalho}

O objetivo visa analisar as relações entre a desrama natural, a dimensão do núcleo enodado e o crescimento. O crescimento de uma árvore é determinado por vários fatores como a qualidade do sítio, espaço vital e a genética, entre outros. Destes resultam o crescimento em diâmetro e altura, que, por sua vez, influenciam a dinâmica e qualidade da desrama natural (Figura 1).

Segundo a hipótese, o crescimento em diâmetro de uma árvore está estreitamente ligado à expansão da sua copa. A altura da inserção da copa depende da produtividade do sítio, expressa pelo crescimento em altura numa determinada idade, e a densidade do povoamento, expressa pelo DAP alcançado. Espera-se que a altura do primeiro galho morto, portanto, esteja estreitamente ligada com a dinâmica da inserção da copa. Assim, como primeiras hipóteses deste trabalho, determina-se:

inserção da copa $=\mathrm{f}(\mathrm{DAP}, \mathrm{ht}, \mathrm{i})$

altura comercial (primeiro galho morto) $=\mathrm{f}$ (inserção da copa)

onde: $\mathrm{DAP}=$ diâmetro na altura do peito $(1,3$ $\mathrm{m}) ; \mathrm{ht}=$ altura total; $\mathrm{i}=$ idade .

A velocidade da expansão da copa determina a galhosidade, ou seja, o núcleo enodado da árvore. A desrama natural, portanto, pode ser influenciada através da condução do crescimento na fase inicial do povoamento. Isso leva à hipótese de que, com as funções acima mencionadas, será possível modelar também a dimensão do núcleo enodado, e, consequentemente, também à parte da madeira sem nós, que representa a parte de mais alto valor do fuste. 


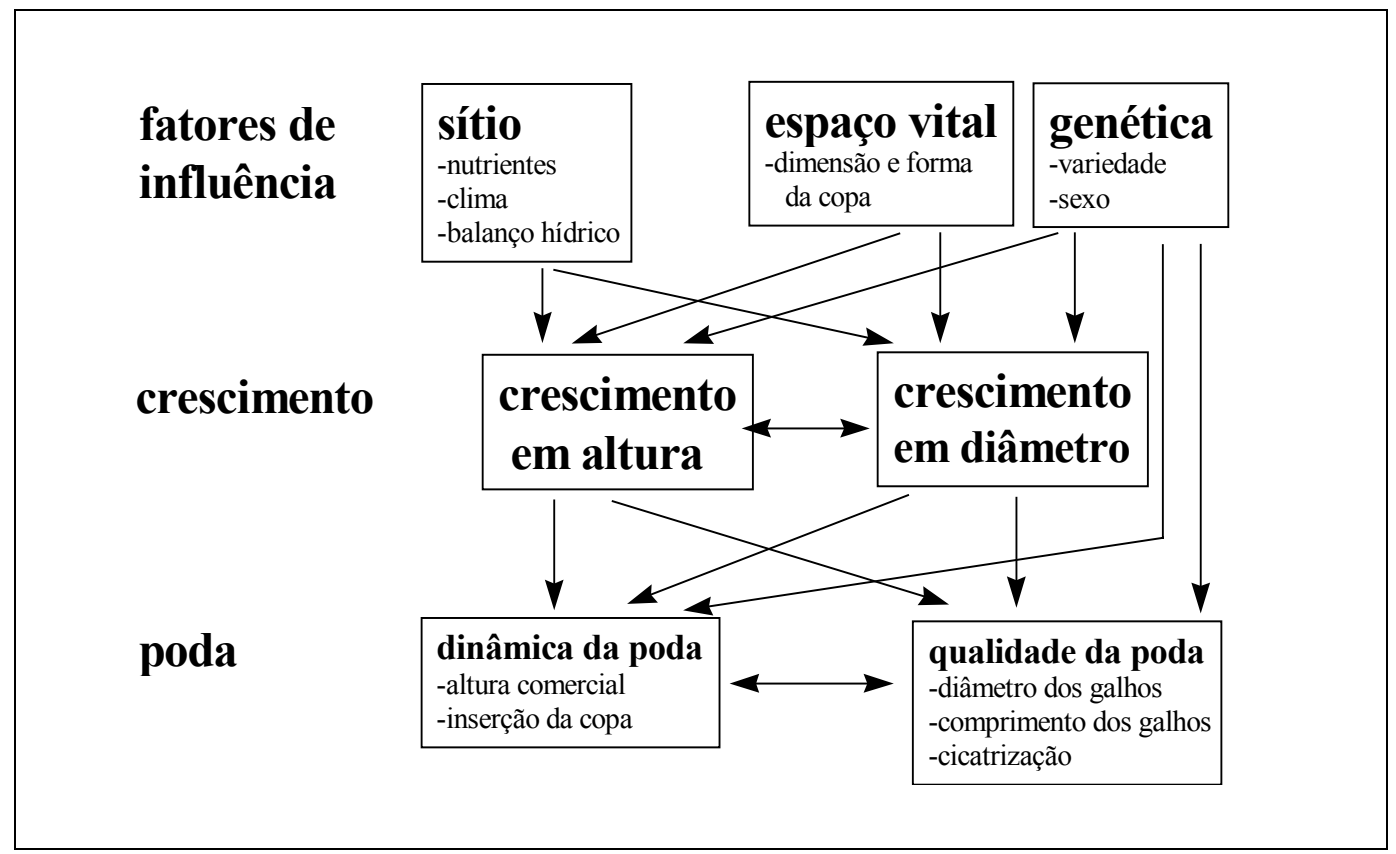

Figura 1: Inter-relações entre os fatores influenciais, o crescimento e a dinâmica, e qualidade da desrama natural

Figure 1: Inter-relationsship between the influencing factors, growth and the dynamics and quality of selfpruning

\section{Área de estudos e povoamento levantados}

As amostras usadas neste estudo se localizam aproximadamente a $29^{\circ} 27^{\circ} \mathrm{Sul}$ e a $50^{\circ} 25^{`}$ Oeste, na Floresta Nacional de São Francisco de Paula (RS), a $27^{\circ} 34^{\prime}$ Sul e a $50^{\circ} 22^{\prime}$ Oeste, nas áreas da empresa CELUCAT em Correia Pinto (SC) e a $25^{\circ} 28^{\prime}$ Sul e a $52^{\circ} 54^{\prime}$ Oeste, nas florestas da empresa ARAUPEL em Quedas do Iguaçu (PR) no ano 2000.

Os tratamentos aplicados nos plantios variam entre sem intervenção, desbaste seletivo e desbaste sistemático. Os espaçamentos iniciais dos plantios foram $2 \mathrm{x}$ $1,5 \mathrm{~m}, 2 \times 2 \mathrm{~m}$ e $3 \times 2 \mathrm{~m}$. Além disso, foram feitas também medições em florestas nativas dominadas por araucária. $\mathrm{Na}$ Tabela 1 são mostrados os valores mínimos e máximos dos parâmetros analisados.

\section{Seleção da amostra, medições e estatística}

Os dados de campo foram utilizados para uma análise exploratória de dados levantados por parcelas temporárias. Para obter informações sobre a competição, fez-se o levantamento de várias árvores numa unidade de amostra. Um estudo preliminar analisando a variação das variáveis mostrou que um número de 25 árvores por amostra é suficiente para obter resultados representativos e estatisticamente seguros. 
Tabela 1: Valores mínimos e máximos dos parâmetros medidos em povoamentos de Araucaria angustifolia na região sul do Brasil

Table 1: Minimum and maximum values of the measured parameters in stands of Araucaria angustifolia in the south of Brazil

\begin{tabular}{|c|c|c|}
\hline PARÂMETRO & MÍNIMO & MÁXIMO \\
\hline $\mathrm{DAP}[\mathrm{cm}]$ & 4,9 & 128,3 \\
\hline idade [anos] & 7 & $49 *$ nas matas nativas mais velhas \\
\hline incremento radial médio [mm/ano] & 1,2 & 11,5 \\
\hline altura do primeiro galho morto [m] & 0,8 & 28,2 \\
\hline altura na inserção da copa $[\mathrm{m}]$ & 1,8 & 32,0 \\
\hline altura total $[\mathrm{m}]$ & 4,9 & 38,4 \\
\hline área de projeção da copa $\left[\mathrm{m}^{2}\right]$ & 0,7 & 306 \\
\hline $\begin{array}{l}\text { índice de sítio }\left(\mathrm{h}_{100} \text { na idade de referência, }\right. \\
\text { SCHNEIDER \& OESTEN 1999) }\end{array}$ & 13 & 32 \\
\hline
\end{tabular}

Os resultados foram interpretados estatisticamente, por meio da análise de correlação e de regressão "stepwise" usando o software SAS ® na versão 6.12.

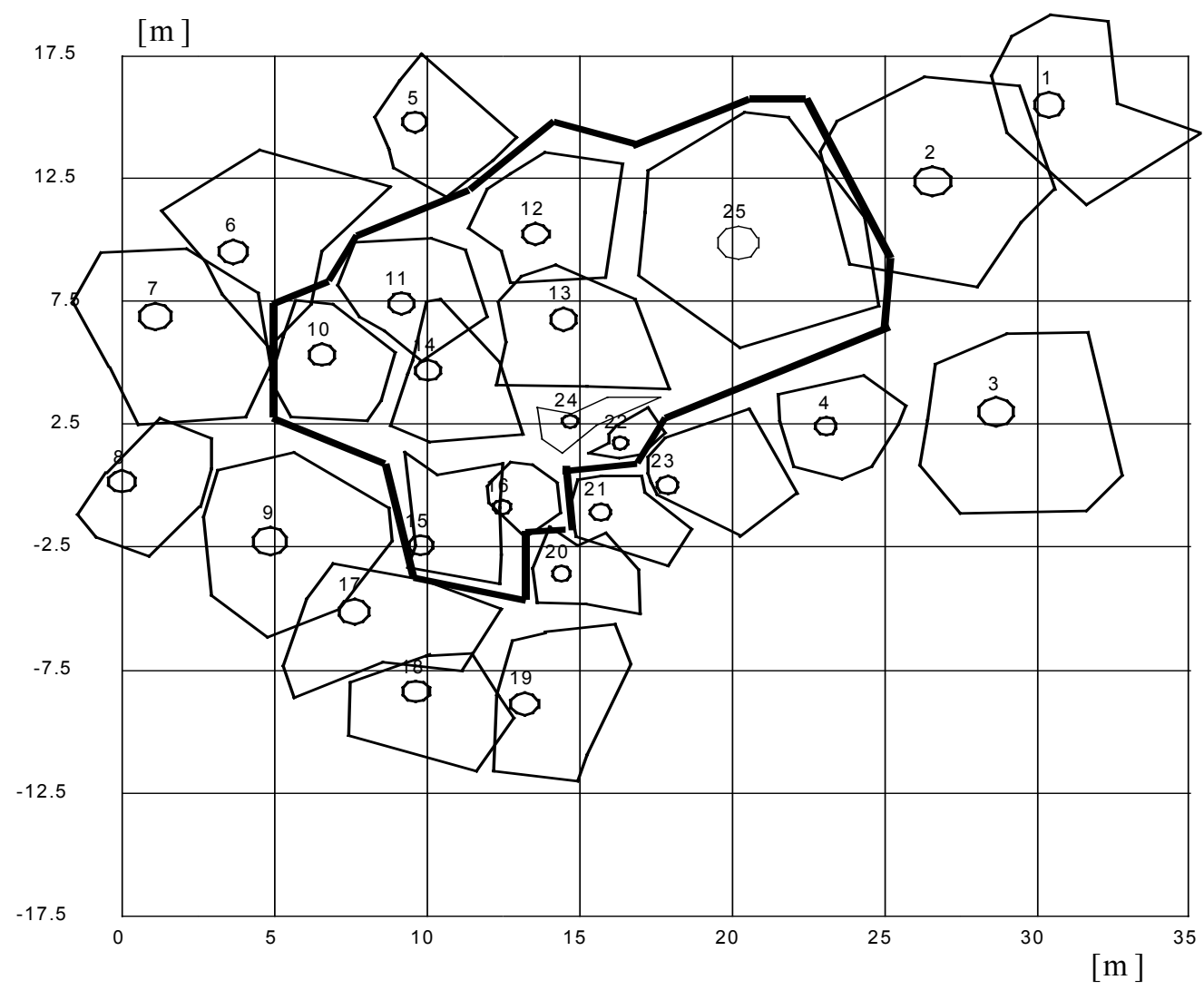

Figura 2: Mapa de distribuição das árvores com as projeções das copas. Através da área destacada (simbólica), foi calculada a percentagem da cobertura das árvores dominantes

Figure 2: Stem distribution map with the crown projection areas. Crown covering percentage of the dominant trees was calculated within the emphasized area

A primeira árvore de cada unidade de amostra foi escolhida ao acaso, com a ajuda de um ângulo e uma distância gerados por computador. A partir desta árvore, para a qual foram dadas as coordenadas $(0 / 0)$, foram localizadas as outras 24 árvores através do ângulo de desvio do Norte e da distância. Em cada árvore foram medidos o DAP, a altura do primeiro galho morto, a altura da inserção da copa e a altura total. A projeção da copa foi determinada através da medição de oito raios com ângulos variáveis, um método 
recomendado para levantamentos em árvores de copas assimétricas (Huber e Röhle, 1985; Röhle, 1986; Spiecker, 1991). Com estes dados foram gerados mapas de distribuição e de projeção da copa (Figura 2).

Dos polígonos obtidos da projeção da copa foi calculada a área, através do método triangular (Röhle, 1986). Em seguida, uma parte das árvores de cada amostra foi destacada com uma linha desenhada à mão, e a área deste polígono foi calculada, com a ajuda de um digitalizador. Da diferença entre este polígono e a soma das áreas das copas dentro do mesmo foi calculada a percentagem da cobertura do dossel. Este valor é de grande importância, pois nem toda área à disposição das árvores é usada pelas copas. Sempre existe uma área sem produtividade, devido a vários fatores (Spiecker, 1991; Nutto, 1999), que devem ser considerados quando se usa modelos espaciais dependentes da distância.

Além da medição da projeção da copa em cada árvore, foram levantados os parâmetros seguindo as hipóteses de trabalho: o diâmetro à altura do peito (DAP), a altura total (ht), a inserção da copa (hic) e a altura comercial (hc). Como inserção da copa foi definido o primeiro galho vivo primário, como altura comercial à altura do primeiro galho morto ainda não nodado. A idade das araucárias foi obtida a partir da data de plantio. Nos povoamentos artificiais, também foram consideradas as classes sociais, segundo Kraft (1884) (Tabela 2). Essa classificação foi feita principalmente para florestas homogêneas e equiâneas e não é totalmente apropriada para árvores em florestas heterogêneas inequiâneas.

Tabela 2: Classe social de árvores segundo a classificação de KRAFT

Table 2: Social position of the trees according to the classification of KRAFT

\begin{tabular}{cl}
\hline Classe & Descrição \\
\hline 1 & árvores pré-dominantes \\
2 & árvores dominantes \\
3 & árvores co-dominantes (intermediárias) \\
4 & árvores dominadas \\
5 & árvores (completamente) suprimidas com copas vivas ou quase mortas \\
\hline
\end{tabular}

Foram também determinados o sexo das árvores (onde foi possível), o estado da copa (vitalidade em classes 1 a 3) e a forma da copa, segundo Seitz (1986).

\section{RESULTADOS E DISCUSSÃO}

\section{Altura da Inserção da copa}

A dinâmica da desrama natural pode ser medida na deslocação da inserção da copa e da altura do primeiro galho morto para cima. Com base na hipótese de trabalho, pode-se concluir que existe uma correlação entre o crescimento em altura e em diâmetro, numa idade determinada e a inserção da copa. A altura numa idade determinada é freqüentemente usada para determinar a qualidade do sítio, enquanto o diâmetro alcançado nesta idade dá informações sobre o crescimento radial. Todas as variáveis estudadas apresentam uma correlação significativa entre si, a um nível de $1 \%$ de probabilidade de confiança (Tabela 3 ).

Tabela 3: Coeficientes de correlação de Pearson (Prob $>|\mathrm{R}| \operatorname{com} \mathrm{H} 0$ : $\mathrm{Rho}=0 / \mathrm{N}=388$ )

Table 3: Pearson correlation coefficients (Prob $>|R|$ com HO: Rho $=0 / N=388$ )

\begin{tabular}{lccccc}
\hline & hic & hc & ht & i & DAP \\
\hline hic & & 0,911 & 0,913 & 0,751 & 0,772 \\
hc & 0,911 & & 0,868 & 0,722 & 0,788 \\
ht & 0,913 & 0,868 & & 0,641 & 0,881 \\
i & 0,751 & 0,722 & 0,641 & & 0,655 \\
DAP & 0,772 & 0,788 & 0,881 & 0,655 & \\
\hline
\end{tabular}

Em que: hic = altura inserção da copa; hc = altura comercial; ht = altura total; i = idade; DAP = diâmetro à altura do peito.

O modelo para estimar a dinâmica da desrama é o seguinte: 


$$
\text { hic }=-2,585+0,12 * i+0,781 * \text { ht }-0,101 * \text { DAP }
$$

Os parâmetros estimados são apresentados na Tabela 4.

Tabela 4: Parâmetros estimados e teste de multicolinearidade

Table 4: Estimated parameters and multicollinearity test

\begin{tabular}{lccccc}
\hline Variável & $\begin{array}{c}\text { parâmetro } \\
\text { estimado }\end{array}$ & Erro padrão & $\begin{array}{c}\text { T para H0: } \\
\text { parâmetro=0 }\end{array}$ & Prob $>|\mathbf{T}|$ & $\begin{array}{c}\text { Inflação da } \\
\text { variância }\end{array}$ \\
\hline intercept & $-2,585$ & 0,2497 & $-10,352$ & 0,0001 & 0,00000 \\
i & 0,120 & 0,0082 & 14,675 & 0,0001 & 1,8092 \\
ht & 0,781 & 0,2906 & 26,893 & 0,0001 & 4,6336 \\
DAP & 0,101 & 0,1302 & $-7,745$ & 0,0001 & 4,7752 \\
\hline
\end{tabular}

Em que: $\mathrm{i}=$ idade; $\mathrm{ht}=$ altura total; DAP $=$ diâmetro à altura do peito.

Para a regressão linear múltipla com hic como variável dependente e a altura, o DAP e a idade como variáveis independentes, foi feita uma análise de variância (Tabela 5). Com uma probabilidade de erro muito pequena

Tabela 5: Análise de variância

Table 5: Variance analysis

\begin{tabular}{lccccc}
\multicolumn{1}{c}{ FV } & GL & SQ & QM & F & Prob $>$ F \\
\hline Modelo & 3 & 7495,156 & 2498,385 & 1109,402 & 0,0001 \\
Erro & 384 & 864,772 & 2,252 & & \\
Total & 387 & 8359,928 & & & \\
\hline
\end{tabular}

Em que: $\mathrm{FV}$ = fonte de variação; $\mathrm{Gl}$ = graus de liberdade; $\mathrm{SQ}$ = soma dos quadrados; $\mathrm{QM}$ = quadrado médio; $\mathrm{F}$ = estatística F de Snedecor.

As variáveis independentes são incluídas no modelo com um alto nível de significância, aumentando a parte da variância explicada pelo modelo. Outras variáveis

Tabela 6: Resultados da regressão 'stepwise' para a variável hic

Table 6: Results of stepwise regression for the variable hic

\begin{tabular}{cccccc}
\hline passo & variável & $\mathbf{R}^{\mathbf{2}}$ (parcial) & $\mathbf{R}_{\text {aju }}{ }^{2}$ modelo) & F & Prob $>\mathbf{F}$ \\
\hline $\mathbf{1}$ & Ht & 0,8337 & 0,8337 & 1934,75 & 0,0001 \\
$\mathbf{2}$ & I & 0,0467 & 0,8804 & 150,40 & 0,0001 \\
$\mathbf{3}$ & DAP & 0,0162 & 0,8966 & 59,98 & 0,0001 \\
\hline
\end{tabular}

Um problema freqüente que se encontra em regressões múltiplas é a correlação entre as variáveis independentes, conhecida como colinearidade ou multicolinearidade (Backhaus, 1990; Hartung e Epelt, 1984). A multicolinearidade pode causar uma seleção aleatória das variáveis no método 'stepwise', porque uma variável pode explicar quase a mesma variação que a outra. Com o aumento da multicolinearidade, corre-se o risco de aumentar desvio padrão dos coeficientes da regressão, deixando a estimativa com baixa confiança. Modificando testadas, tais como DAP ${ }^{2}, \mathrm{ht}^{*} \mathrm{DAP}, \mathrm{ht}^{2}, \mathrm{i}^{2}$, $i^{*}$ DAP e $i * h t$ não diminuíram a variação do modelo (Tabela 6).
(Prob $>\mathrm{F}=0,0001)$, a relação entre as variáveis é significativa e o modelo pode ser utilizado para a estimativa da hic. O coeficiente de determinação ajustado foi igual a 0,896. 
mostraram estáveis quando a base de dados foi mudada aleatoriamente. O exame dos resíduos mostrou uma homogeneidade das variâncias dos valores de yi dentro das classes de xi.

O modelo também é consistente considerando os fatores biológicos que as variáveis representam. Ambas, a altura e a idade, têm um sinal positivo, quer dizer, com o aumento destes valores o parâmetro estimado também aumenta. Por outro lado, um DAP maior numa idade determinada significa uma inserção da copa mais baixa, porque o coeficiente deste parâmetro é negativo. A conseqüência lógica é que as árvores mais grossas de um povoamento, geralmente, mostram copas com inserção mais baixa. Isso significa que, controlando a competição, o crescimento em diâmetro pode ser manejado, e com este também a dinâmica da desrama natural.

\section{Altura comercial}

A correlação estreita entre a inserção da copa e a altura comercial foi usada para modelar a mesma. Numa simples regressão

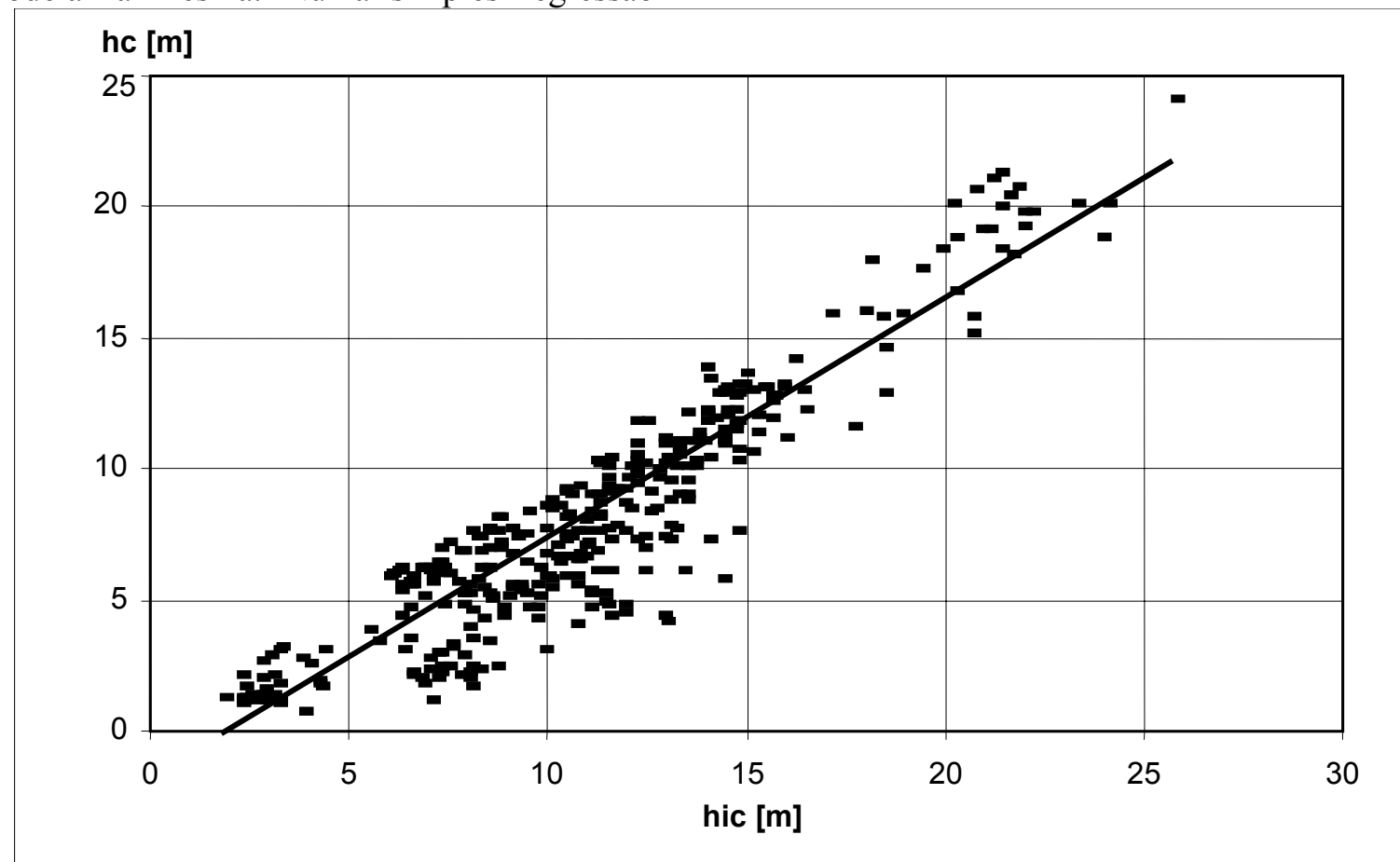

Figura 3: Altura comercial em função da inserção da copa de árvores de Araucaria angustifolia. Dez valores foram classificados como 'outliers' e foram eliminados

Figure 3: Height of lowest dead branch as a function of height of crown base of Araucaria angustifolia. Ten values were classified as outliers and eliminated

Tabela 7: Parâmetros do modelo para estimar a altura comercial 
Table 7: $\quad$ Model parameters for estimating height of lowest dead branch

\begin{tabular}{lcccc}
\hline Variável & $\begin{array}{c}\text { parâmetro } \\
\text { estimado }\end{array}$ & Erro padrão & $\begin{array}{c}\text { T para H0: } \\
\text { parâmetro=0 }\end{array}$ & Prob $>|\mathbf{T}|$ \\
\hline intercept & $-1,957$ & 0,2424 & $-8,074$ & 0,0001 \\
hic & 0,919 & 0,0211 & 43,518 & 0,0001 \\
\hline
\end{tabular}

\section{Aplicação dos modelos para estimar a dinâmica da desrama natural}

Para a dinâmica da desrama natural, destacam-se dois fatores de maior influência: o crescimento em diâmetro e em altura. Geralmente o crescimento em altura depende da qualidade do sítio e é muito difícil de manejar. $\mathrm{O}$ crescimento em diâmetro, pelo contrário, pode ser regulado facilmente através de desbastes.

A Figura 4 mostra a diferença na desrama natural em duas árvores crescendo no mesmo sítio, mas com diferentes crescimentos radiais. $\mathrm{O}$ crescimento em diâmetro pode ser manejado através da largura da copa (Nutto,
2001). As árvores com copas maiores mostram uma desrama natural lenta, quer dizer galhos com inserção mais baixa. Um crescimento em diâmetro forçado por desbastes ou maiores espaçamentos tem como conseqüência uma galhosidade maior.

Como mostra a Figura 5, existe uma grande diferença na desrama natural, causada somente pelo crescimento em altura, mesmo que o crescimento em diâmetro seja igual. Isso significa que, para obter a mesma dinâmica e qualidade da desrama natural em sítios diferentes, é necessário diminuir o crescimento em diâmetro no sítio com qualidade mais alta.

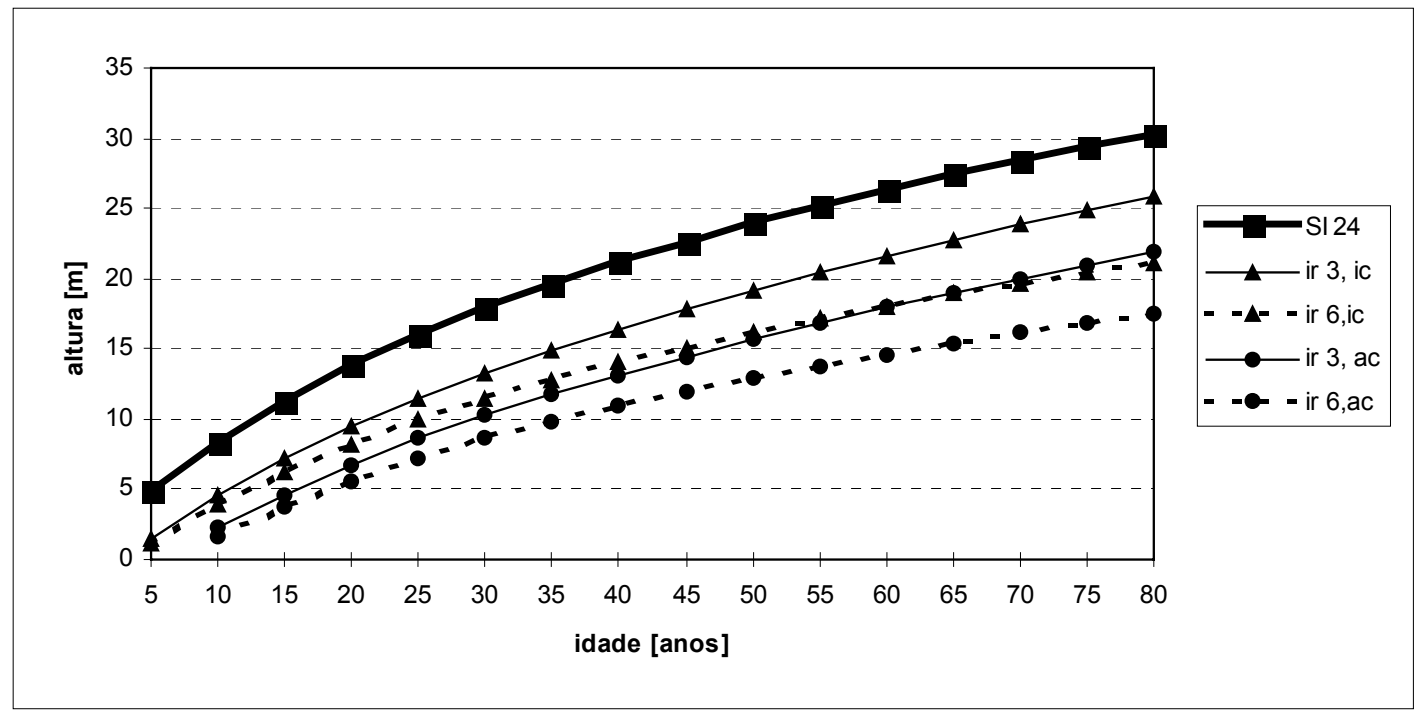

Figura 4: Comparação da influência do crescimento em diâmetro na desrama natural. A curva hipsométrica (SI 24, tabelas de produção de Schneider e Oesten, 1999), a altura da inserção da copa e a altura comercial dependem do crescimento radial (3 e $6 \mathrm{~mm} / \mathrm{ano})$

Figure 4: Comparison of the influence of diameter growth on self-pruning. Height (SI 24, yield tables of Schneider and Oesten, 1999), height of crown base and height of lowest dead branch depend from radial increment (3 and $6 \mathrm{~mm} /$ year). 


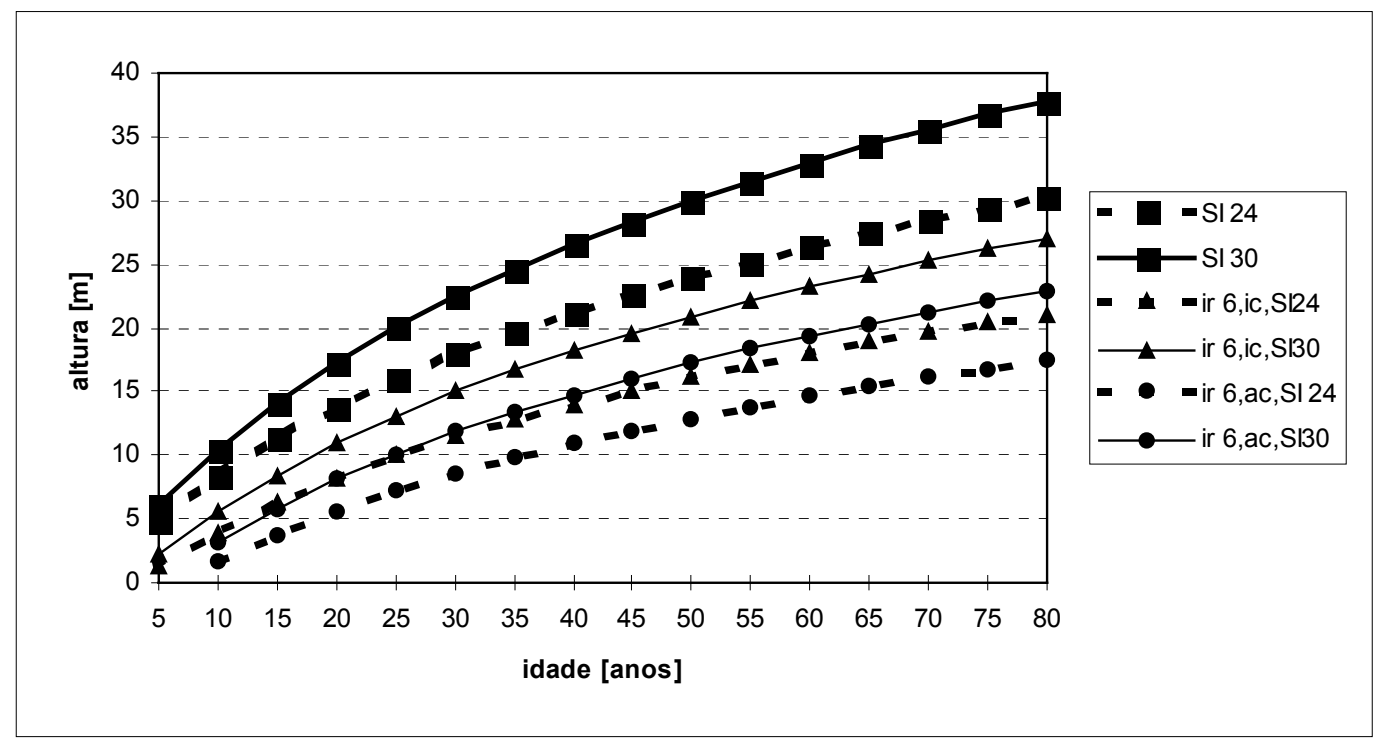

Figura 5: Influência do sítio (SI 24 e 30 das tabelas de produção de Schneider e Oesten, 1999) na desrama natural de Araucaria angustifolia (crescimento radial $=6 \mathrm{~mm} / \mathrm{ano}$ ).

Figure 5: Influence of site (SI 24 and 30 of the yield tables of Schneider and Oesten, 1999) on self-pruning of Araucaria angustifolia (radial increment $=6 \mathrm{~mm} /$ year)

\section{Núcleo enodado}

Com as informações obtidas dos modelos da dinâmica da desrama, pode-se calcular a dimensão do núcleo enodado, na dependência do crescimento em altura e em diâmetro. No exemplo, o crescimento em altura segue as curvas hipsométricas da tabela de produção de Schneider e Oesten (1999). O crescimento em diâmetro exprime-se em diferentes crescimentos radiais. 


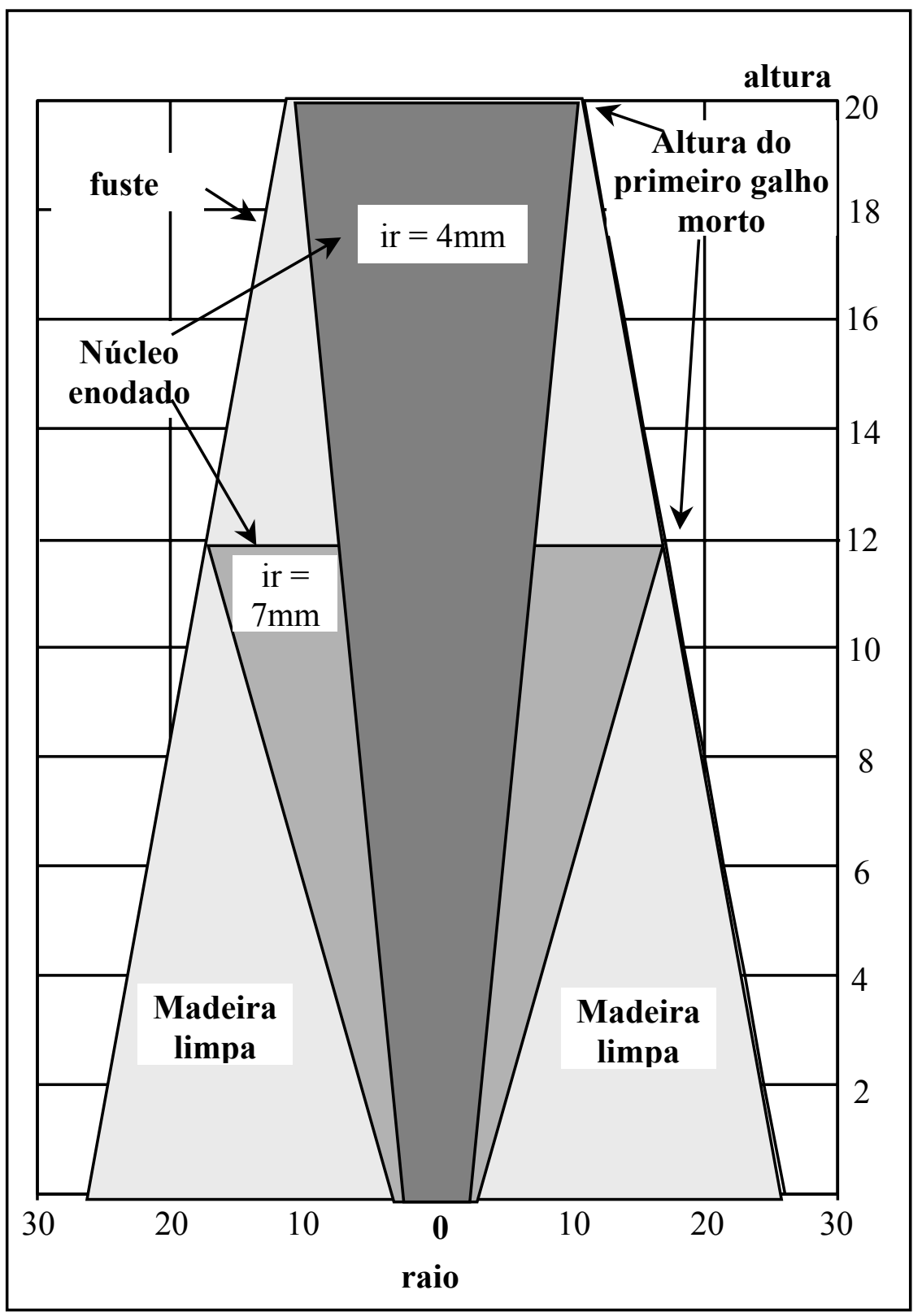

Figura 6: Modelo para estimar a qualidade interna do tronco com nós para Araucaria angustifolia, para um crescimento radial de 4 e $7 \mathrm{~mm} /$ ano, uma conicidade de $3 \%$ por metro corrido, um diâmetro-objetivo de $50 \mathrm{~cm}$ e um crescimento em altura segundo o índice de sítio 30 (tabelas de produção Schneider e Oesten, 1999)

Figure 6: Model to estimate the internal quality of the stem with knots for Araucaria angustifolia, for a radial increment of 4 and $7 \mathrm{~mm} /$ year, a conicity of $3 \%$ per meter, a goal-diameter of $50 \mathrm{~cm}$ and height growth according to site index 30 (yield tables of Schneider and Oesten, 1999)

Com estes dados, pode ser calculada a altura do primeiro galho morto para um certo crescimento em diâmetro e uma idade determinada. Considerando uma conicidade da araucária de 3\% por metro corrido (Schneider e Oesten, 1999), pode-se calcular a dimensão do núcleo enodado (Figura 6).

Como mostra a Figura 6, a parte da madeira sem nós pode ser aumentada significativamente com um crescimento em diâmetro reduzido. Mas isto também significa uma rotação mais longa para alcançar $\mathrm{o}$ diâmetro-objetivo. No exemplo dado (Figura 6), a diferença é de 27 anos, com uma rotação de 63 anos para a variante de $4 \mathrm{~mm} / \mathrm{ano}$ e de 36 anos para a variante de $7 \mathrm{~mm} / \mathrm{ano}$, 
respectivamente. $\mathrm{O}$ volume do fuste até a altura comercial $(20 \mathrm{~m})$ chega a $3.28 \mathrm{~m}^{3}$ na variante de $4 \mathrm{~mm} / \mathrm{ano}$, com um núcleo enodado de $0,56 \mathrm{~m}^{3}$ e um manto de madeira limpa de $2,7 \mathrm{~m}^{3}$. Na variante de $7 \mathrm{~mm}$, o volume somente chega a $1,97 \mathrm{~m}^{3}$ com uma altura comercial de $12 \mathrm{~m}$, um núcleo enodado de 0,75 $\mathrm{m}^{3}$ e um manto de madeira limpa de $1,22 \mathrm{~m}^{3}$, alcançando, assim, somente $43 \%$ da variante 1.

A parte da madeira valiosa sem nós, principalmente nos primeiros 6 até os 10 metros, pode ser aumentada, se o crescimento em diâmetro for reduzido, quer dizer a competição entre as árvores é mantida por um período mais longo. Isto também significa que o espaçamento inicial deve ser mais denso. A dificuldade é achar um equilíbrio entre os custos para o plantio, a poda artificial, o período de rotação e a dimensão do núcleo enodado, de acordo com as metas de produção estabelecidas. Um manto de madeira sem nós de, no mínimo, 2/3 do diâmetro é desejável para uma utilização da madeira para serraria ou laminação (Schulz, 1959). O mesmo autor confirma que nos primeiros $4 \mathrm{~m}$ do fuste se concentra mais que $60 \%$ do valor da árvore. Esta dimensão do manto de madeira limpa é muito difícil de obter, especialmente com os diâmetros-objetivo relativamente baixos e as rotações de 40 anos, que, geralmente, são aplicadas no manejo de araucária no Sul do Brasil. Por enquanto, para árvores como as coníferas, com uma desrama natural retardada, uma poda artificial é recomendável para aumentar significativamente $o$ valor das árvores.

Com base nas dimensões dos núcleos enodados, calculados através dos modelos (Equações 2 e 3), pode-se avaliar se a desrama natural é suficiente para alcançar as metas desejadas. Se esse não for o caso, deve ser considerada uma poda artificial. $\mathrm{O}$ procedimento mais fácil, sem perda de produção de volume, seria uma poda dos galhos mortos, mantendo assim a altura comercial sempre no nível da inserção da copa.

Tabela 8: Dinâmica da desrama natural de araucária para um sítio do Índice SI 30 e um crescimento radial de 7 $\mathrm{mm} / \mathrm{ano}$

Table 8: Dynamic of self-pruning of araucária for a site of SI 30 and radial growth of $7 \mathrm{~mm} /$ year

\begin{tabular}{|c|c|c|c|c|c|}
\hline Idade & $\begin{array}{l}\text { ht }[\mathrm{m}] \\
(\mathrm{SI} 30)\end{array}$ & $\begin{array}{l}\mathrm{hc} \\
{[\mathrm{m}]}\end{array}$ & $\begin{array}{l}\text { hic } \\
{[\mathrm{m}]}\end{array}$ & $\begin{array}{c}\text { comprimento relativo } \\
\text { da copa }[\%]\end{array}$ & $\begin{array}{c}\text { DAP }[\mathrm{cm}] \\
\text { (ir }=7 \mathrm{~mm} / \mathrm{ano})\end{array}$ \\
\hline 5 & 6,09 & & 2,1 & 66 & 7 \\
\hline 10 & 10,42 & 3,0 & 5,4 & 62 & 14 \\
\hline 15 & 14,12 & 5,5 & 8,2 & 53 & 21 \\
\hline 20 & 17,3 & 7,7 & 10,5 & 47 & 28 \\
\hline 25 & 20,6 & 9,6 & 12,6 & 44 & 35 \\
\hline 30 & 22,47 & 11,3 & 14,4 & 42 & 42 \\
\hline 35 & 24,61 & 12,7 & 16 & 40 & 49 \\
\hline 40 & 26,54 & 14 & 17,4 & 39 & 56 \\
\hline
\end{tabular}

Os valores da tabela 8 mostram que, nos primeiros 10 até 15 anos, quando já cresceram $40 \%$ do diâmetro-objetivo, as árvores mantinham uma boa parte da copa viva. Por isso deve ser considerada também uma poda dos verticílios de galhos ainda vivos quando o comprimento relativo da copa é de $40 \%$ ou mais. Também deve ser considerado que, nesta idade, a dinâmica do crescimento em altura é alta. O crescimento em altura é o melhor critério para determinar a intensidade tolerável de poda de galhos vivos para uma árvore (Hawley e Smith, 1972). A mortalidade dos galhos também está estreitamente relacionada com a dinâmica do crescimento em altura. Assim, o processo da desrama é somente antecipado, sem influenciar negativamente o crescimento em diâmetro em um período longo. Montagna et al. (1993) concluíram que o crescimento em altura não é influenciado pela poda artificial, especialmente nos estágios jovens das árvores.

No exemplo calculado em tabela 8 e mostrado na figura 7, uma poda de até $3 \mathrm{~m}$ na idade de 5 anos e uma segunda na idade de 9 anos de até $6 \mathrm{~m}$ melhorariam 
significativamente a qualidade do fuste neste crescimento em diâmetro seja negativamente período de tempo trecho, sem que o influenciado em um longo período.

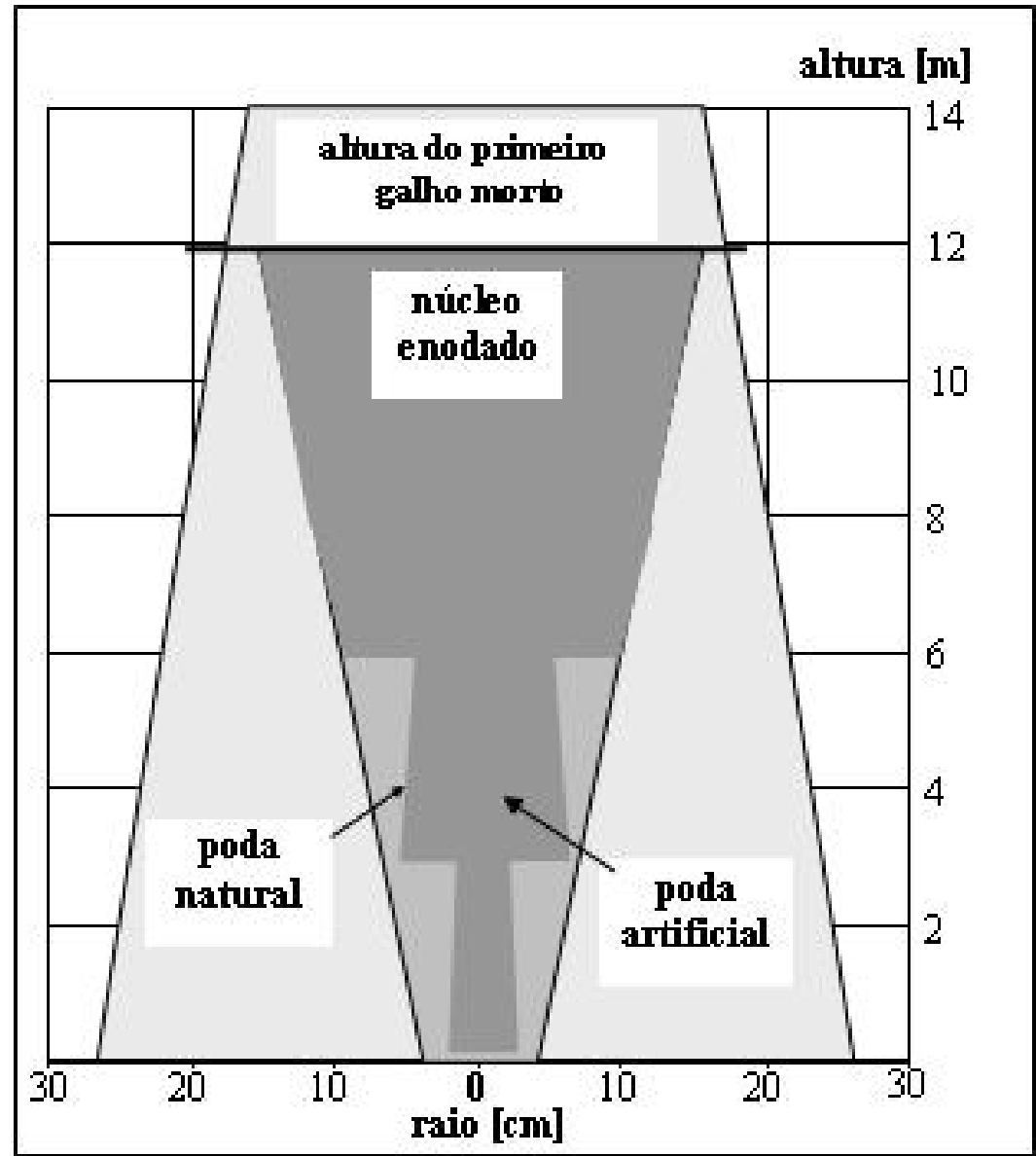

Figura 7: Comparação da desrama natural e artificial (nas idades de 5 e 10 anos até $40 \%$ do comprimento relativo da copa), para um índice de sítio SI 30 (Schneider \& Oesten 1999) e um crescimento radial de $7 \mathrm{~mm} / \mathrm{ano}$

Figure 7: Comparison of natural and arificial pruning (at the age of 5 and 10 years until 40\% of crown ratio), for a SI of 30 (Schneider \& Oesten 1999) and a radial increment of $7 \mathrm{~mm} /$ year

\section{CONCLUSÕES E RECOMENDAÇÕES}

A dinâmica e qualidade da desrama natural de Araucaria angustifolia foram modeladas com ajuda das variáveis DAP, altura e idade. Os fatores que influenciam este processo são o crescimento em altura $\mathrm{e}$ diâmetro. Já que o crescimento em altura somente pode ser influenciado com um manejo intensivo e caro, o crescimento em diâmetro pode ser manejado muito mais facilmente e com alta eficiência sobre o regulamento da competição entre as árvores, quer dizer desbastes. Enquanto um crescimento em altura elevado causa uma desrama natural acelerada, um crescimento em diâmetro forçado retarda este processo. Por causa disto, obtém-se uma melhor desrama natural nos sítios de alta qualidade, com o mesmo crescimento em diâmetro, comparado com um sítio de baixa qualidade. Por outro lado, consegue-se melhorar a dinâmica da desrama mantendo os povoamentos fechados. Com isto, o crescimento em diâmetro também é menor, o que significa um período de rotação mais longo.

A qualidade da madeira em relação ao núcleo enodado de araucária foi modelada, usando os modelos da dinâmica da desrama. Assim, pode-se facilmente calcular a eficiência de uma desrama natural para diferentes crescimentos em diâmetro, considerando também a qualidade do sítio. Para a araucária que possui uma dinâmica de desrama natural lenta, é recomendável aplicar uma poda 
artificial com o objetivo de melhorar a qualidade da madeira, especialmente nas rotações curtas e diâmetros-objetivo de 40 até $50 \mathrm{~cm}$.

Enfim, também deve ser considerada a possibilidade de aumentar os diâmetrosobjetivo das árvores, se a meta de produção for madeira de alta qualidade. O núcleo enodado, depois de ser formado, é um fator constante, enquanto a madeira que cresce ao redor do fuste limpo aumenta continuamente.

\section{AGRADECIMENTOS}

Agradecemos a Saulo José Barbieri, Eng $^{\circ}$ Florestal e Chefe do Setor de Planejamento Florestal, e a Vilmar de Souza Brasil, Técnico, ambos da empresa ARAUPEL S.A, Quedas do Iguaçu, PR.

Este trabalho foi realizado com a ajuda do programa de pós-doutorado HSP III do Governo Federal da Alemanha, sob coordenação do DAAD.

\section{REFERÊNCIAS}

BACKHAUS, K. Multivariate Analysemethoden: eine anwendungsorientierte Einführung. 6.Auflage. Berlin und Heidelberg, Springer, $1990.416 \mathrm{p}$.

BELSLEY, D. A. et al. Regression diagnostics: identifying influential data sources of collinearity. New York, John Willey \& Sons, 1980. 292 p.

DE HOOG, R. J. Site-nutrition-growth relationships of Araucaria angustifolia (Bert) O. Ktze. in southern Brazil. Dissertation an der Universität Freiburg, Freiburg 1981. 161 p.

DELEUZE, C.; HERVE, J-C.; COLIN, F.; Ribeyrolles, L. Modelling crown shape of Picea abies: spacing effects. Canadian Journal of Forest Research, 26: p. 19571966, 1996.

DRAPER, N. R.; SMITH, H. Applied regression analysis. New York: Wiley, 1966. $407 \mathrm{p}$.

GOLFARI, L. Coniferas aptas para repoblaciones forestales en el Estado de São Paulo. Silvicultura em São Paulo, 6: p.7-62, 1967.
HARTUNG, J.; ELPELT, B. Multivariate Statistik. München, Oldenbourg Verlag, 1984. $806 \mathrm{p}$.

HAWLEY, R. C.; SMITH, D. M. Silvicultura prática. Barcelona: Omega, 1972. 544 p.

HUBER, W.; RÖHLE, H. Untersuchungen zur Methode der Ablotung von Kronenradien und der Berechnung von Kronengrundflächen. Forstarchiv, 56: p.238-243, 1985.

HUECK, K. As florestas da América do Sul. São Paulo: Polígono, 1972. 466 p.

IBAMA. Relatório da Comercialização de espécies contingenciadas. Disponível em: $<\mathrm{http}$ ://www.ibama.gov.br./onda/tabelas/in dice.htm>, 32 tabelas. Accesso em decembro 1999

KOZLOWSKI, T. T. Growth and development of trees. New York: Academic Press, 1971. $443 \mathrm{p}$.

KRAFT, G. 1902. Beiträge zur Lehre von den Durchforstungen, Schlagstellungen und Lichtungshieben (1884). In: Die diesjährige Versammlung des Vereins Forstlicher Versuchsanstalten, von WIMMENAUER. Allgemeine Forst- und Jagdzeitung. p.422425, 1902 .

LAMPRECHT, H. Waldbau in den Tropen. Hamburg und Berlin, Paul Parey Verlag, $1986.318 \mathrm{p}$.

MACHADO, S. A.; SIQUEIRA, J. D. P. Distribuição natural de Araucaria angustifolia (Bert.) O. Ktze. 1980, p. 4-9. Trabalho apresentado na conferência IUFRO "Problemas florestais do gênero Araucaria",1980, Curitiba.

MAYER-WEGELIN, H. Ästung. Hannover, M.\&H. Schaper, 1936. 178 p.

MITSCHERLICH, G.; VON GADOW, K. 1968. Über den Zuwachsverlust bei der Ästung von Nadelbäumen. Allgemeine Forst- und Jagdzeitung, 139 (8). p. 175-185., 1968.

MONTAGNA, R. G.; FERNANDES, P. S.; ROCHA, F. T. et al. Influencia da desrama artificial sobre o crescimento e a densidade básica da madeira de Pinus elliottii var. elliottii. Serie Técnica IPEF, 9 (.27). p. 35-46, 1993. 
NELSON, N. D.; BURK, T. \& ISEBRANDS, J.G. 1981. Crown architecture of short-rotation intensively cultivated Populus. I. Effects of clone and spacing on first-order branch characteristics. Canadian Journal of Forest Research 11 (2). p. 73-81, 1981.

NUTTO, L. Neue Perspektiven in der Begründung und Pflege von jungen Eichenbeständen: Ergebnisse einer Untersuchung zur Kronenentwicklung, Astreinigung und Dickenwachstum junger Stiel- und Traubeneichen in Europa (Quercus robur L. und Quercus petraea (Matt.) Liebl.). Schriftenreihe Freiburger Forstliche Forschung Band 5. Freiburg i. Brsg., 1999. 190p.

NUTTO, L. Manejo do crescimento em diâmetro de Araucaria angustifolia (Bert.) O. Ktze. Baseado na árvore individual. Ciência Florestal, 11 (2). p. 9-21, 2001.

NUTTO, L.; SPIECKER, H. 2000. Production of valuable wood: a software aided decision tool for managing oak stands in Europe. Annales Experimentalis Silvarum Culturae Provehendis, 34, Zagreb, Croatia. p.37-49. Trabalho apresentado na conferência IUFRO "Improvement of wood quality and genetic diversity of oaks", Zagreb, Croatia, Abril 2000.

RÖHLE, H. Vergleichende Untersuchungen zur Ermittlung der Genauigkeit bei der Ablotung von Kronenradien. Forstarchiv, 57. p.67-71, 1986.

SCHILLING, A. C. Influência da desrama sobre a qualidade da madeira de primeiro desbaste de Pinus elliottii Engelm., 1996, 73 p. Dissertação de Mestrado, Universidade Federal de Santa Maria, Santa Maria - RS.

SCHNEIDER, P. R. Análise de regressão aplicada à Engenharia Florestal. 2. Ed. Santa Maria: UFSM, CEPEF, 1998. 236 p.

SCHNEIDER, P. R.; OESTEN, G. Tabelas auxiliares para o manejo de Araucaria angustifolia e Pinus elliottii para a região do planalto médio do Rio Grande do Sul. CEPEF, Universidade Federal de Santa Maria, 1999. $118 \mathrm{p}$.
SCHULZ, H. Untersuchungen über die Bewertung und Gütemerkmale des Eichenholzes aus verschiedenen Wuchsgebieten. Schriftenreihe der Universität Göttingen, 23, 1959. 90 p.

SEITZ, R. Erste Hinweise für die waldbauliche Behandlung von Araukarienwäldern. Annales des Sciences Forestieres, 43. p.327-338, 1986.

SHIGO, A. L. Tree decay: An expanded concept. USDA Fo.Ser.Agric.Inf.Bull. No. 419, 1979. $73 \mathrm{p}$.

SPIECKER, H. Zur Steuerung des Dickenwachstums und der Astreinigung von Trauben- und Stieleichen. Schriftenreihe der Landesforstverwaltung B.-W., 72, 1991. 155 p.

WHITMORE, T. C. Tropical rain forests of Far East, Oxford, 1975. 352 p. 